Bull. Austral. Math. Soc.

20F05, 20D15

VOL. $62(2000) \quad[467-469]$

\title{
AN INEQUALITY FOR GROUP PRESENTATIONS
}

\author{
Avionam Mann
}

\section{To Bernhard Neumann on his 90th birthday}

\begin{abstract}
We prove an inequality involving the number of relations and of generators of any group, and derive some consequences. The proof applies the theory of pro- $p$ groups.
\end{abstract}

Let a group $G$ be presented by $b$ generators and $r$ relations. The difference $r-b$ (or, alternately, $b-r$ ) is known as the deficiency of the presentation. It seems that Bernhard Neumann was the first to draw attention explicitly to this quantity [4]. We denote by $G^{\prime}$ the commutator (derived) subgroup of $G$, and $\gamma_{3}(G)=\left[G^{\prime}, G\right]$ is the next term of the lower central series. $G^{n}$ denotes the subgroup generated by the $n$th powers. Fix a prime $p$, and denote $\Phi^{1}=\Phi^{1}(G)=G^{\prime} G^{p}$ and $\Phi^{2}=\Phi^{2}(G)=\left[\Phi^{1}(G), G\right] \Phi^{1}(G)^{p}$. Define integers $d=d_{p}(G)$ and $e=e_{p}(G)$ by $\left|G / \Phi^{1}\right|=p^{d_{p}(G)}$ and $\left|\Phi^{1} / \Phi^{2}\right|=p^{e_{p}(G)}$.

If $p>2$, write also $\Psi=\Psi(G)=\left[\Phi^{1}, G\right] G^{p}\left(=\gamma_{3}(G) G^{p}\right)$ and $\left|\Phi^{1} / \Psi\right|=p^{f_{p}(G)}=$ $p^{f}$. For $p=2$, define $f$ by first replacing $G^{p}$ by $G^{4}$ in the definitions of $\Phi^{1}$ and $\Psi$, and then taking $f_{2}(G)$ to be the minimal number of generators of $\Phi^{1} / \Psi$.

THEOREM. $r-b \geqslant d^{2} / 2-d / 2-e$.

Corollary $1 . r-b \geqslant d^{2} / 2-3 d / 2-f$.

If $G$ is a finite $p$-group, one can take $b=d$. In that case, the inequality of the theorem (for $p=2$ ) or of the corollary (for odd $p$ ) is one of several that were proved for $p$-groups in [3]. The point of the present note is that these inequalities hold without any restriction on $G$.

ProOF OF THEOREM: We consider the given presentation as a presentation in the variety of pro- $p$ groups. It then defines the pro- $p$ completion $\widehat{G}$ of $G$ (for elementary facts about pro- $p$ groups, see, for example [2]). Since $G$ and $\widehat{G}$ have the same finite $p$-groups as homomorphic images, it follows that $d_{p}(G)=d_{p}(\widehat{G})$ and $e_{p}(G)=e_{p}(\widehat{G})$.

Received 4th April, 2000

This note was written while the author was enjoying the hospitality of the School of Mathematical Sciences and The Australian National University, Canberra, and of the Department of Mathematics at the University of Western Australia, Perth.

Copyright Clearance Centre, Inc. Serial-fee code: 0004-9727/00 \$A2.00+0.00. 
But $\Phi^{1}(\widehat{G})$ is the Frattini subgroup $\Phi(\widehat{G})$ of $\widehat{G}$, so $\widehat{G}$ is generated (as a pro- $p$ group) by $d$ elements. Choose $e$ elements of $G$ that generate $\Phi^{1}\left(\operatorname{Mod} \Phi^{2}\right)$, write them as words in the given generators of $G$, and add them to the set of relators of $\widehat{G}$. We obtain a pro- $p$ group $H$ with $r+e$ relations satisfying $\Phi^{1}(H)=\Phi^{2}(H)=\left[\Phi^{1}(H), H\right] \Phi^{1}(H)^{p}$. In a pro- $p$ group, as in a finite $p$-group, such an equality implies $\Phi^{1}(H)=1$, that is, $H$ is an elementary Abelian group of order $p^{d}$. But this group cannot be defined on $b$ generators by less than $d^{2} / 2+d / 2+b-d$ relations, hence our inequality.

The corollary follows by combining the theorem with the following

PROPOSITION $1 . e \leqslant d+f$.

ProOF: Note that $e$ is the minimal number of elements needed to generate $\Phi^{\mathbf{1}}(\widehat{G})$ as a normal subgroup of $\widehat{G}$. Take $f$ elements that generate $\Phi^{1}(\operatorname{Mod} \Psi)$ and add them to the relators of $\widehat{G}$, to obtain a pro- $p$ group $K$, which is the quotient of $\widehat{G}$ by the normal closure of the $f$ elements. Then $\Phi^{1}(K)=\Psi(K)$, and thus $K^{\prime} \leqslant\left[K^{\prime}, K\right] K^{p}$. This implies that $K^{\prime} \leqslant K^{p}$ (for $p=2, K^{\prime} \leqslant K^{4}$ ), and that means that $K$ is a so called powerful pro- $p$ group (see [2, Chapter 3]). Then $K^{p}$ is also generated by $d$ elements [2, Theorem 3.6(iii)], and preimages in $G$ of these generators, together with the $f$ generators of $\Phi^{1} / \Psi^{2}$, constitute a set of normal generators for $\Phi^{1}$. Therefore $e \leqslant d+f$.

Corollary 2. Let $G$ be defined by $b$ generators and $r$ relations, let $t_{0}$ and $s_{0}$ be the torsion free ranks of $G / G^{\prime}$ and of $G^{\prime} / \gamma_{3}(G)$, respectively, and let $t_{p}$ and $s_{p}$ be the minimal numbers of generators of the Sylow $p$-subgroups of the same factor groups. Then the inequality of Corollary 1 holds for the values $d=t_{0}$ and $f=s_{0}$, and also for $d=t_{0}+t_{p}$ and $f=s_{0}+s_{p}$.

Proof: We have $d_{p}(G)=t_{0}+t_{p}$, and since $\Phi^{1} / \Psi \cong G^{\prime} /\left(G^{\prime} \cap \Psi\right)$, and the latter group is an image of $G^{\prime} / \gamma_{3}(G)$, also $f_{p} \leqslant s_{0}+s_{p}$. Moreover, since $G / G^{\prime}$ and $G^{\prime} / \gamma_{3}(G)$ are finitely generated Abelian groups, there exists a prime $p$ such that $t_{p}=s_{p}=0$. [

If $r<b$ the theorem implies that $d>0$ (because if $d=0$ then $G=\Phi_{1}=\Phi_{2}$, so also $e=0$ ). Indeed it is well known that in that case $G$ has an infinite cyclic factor group (simply consider the given presentation as one in the variety of Abelian groups; it then defines the Abelianisation $G / G^{\prime}$ ). If $b \geqslant r+2$ we get even that $f>0$, so $G$ has non-Abelian $p$-factor-groups. This is also known: in [1, p.73, Lemma 5] it is shown that in that case $G$ has a quotient isomorphic to the wreath product of a group of order $p$ by an infinite cyclic group.

Finally we note that in the proof of Proposition 1 we have constructed a powerful quotient group of $\widehat{G}$. It turns that this is the only way to find such quotient groups.

Proposition 2. Let $G$ be a pro-p group, and let $N \triangleleft G$. Then $G / N$ is powerful if and only if $N$ contains $f$ elements generating $\Phi(G)(\operatorname{Mod} \Psi(G))$. 
Proof: We have to prove only the 'only if' part. So let $G / N$ be powerful. That means that $G^{\prime} \leqslant N G^{p}$, and then also $\Phi:=\Phi(G)=G^{\prime} G^{p} \leqslant N G^{p}$, and therefore $\Phi=(N \cap \Phi) G^{p}$. Similarly $\Psi=(N \cap \Psi) G^{p}$. Thus any set of generators for $(N \cap \Phi)(\operatorname{Mod}(N \cap \Psi))$ is also a set of generators for $\Phi(\operatorname{Mod} \Psi)$. Moreover, $\Phi / \Psi \cong(N \cap \Phi) /(N \cap \Phi) \cap(N \cap \Psi) G^{p}=(N \cap \Phi) /(N \cap \Psi)$. This factor group is elementary Abelian of order $p^{f}$, so any set of generators for it contains a subset of $f$ generators.

\section{REFERENCES}

[1] G. Baumslag, Topics in combinatorial group theory (Birkhaüser, Basel, 1993).

[2] J.D. Dixon, M.P.F. du Sautoy, A. Mann and D. Segal, Analytic pro-p groups, 2nd ed. (Cambridge University Press, Cambrdige, 1999).

[3] W. Gaschütz and M.F. Newman, 'On presentations of finite p-groups', J. Reine Angew. Math. 245 (1970), 172-176.

[4] B.H.Neumann, On some finite groups with trivial multiplicator, Publ. Math. 4 (1956), 190-194.

Einstein Institute of Mathematics

The Hebrew University of Jerusalem

Givat Ram

91904 Jerusalem

Israel

e-mail: mann@math.huji.ac.il 